\title{
BMJ Open Transcultural mediation programme in a paediatric hospital in France: qualitative and quantitative study of participants' experience and impact on hospital costs
}

\author{
Jonathan Lachal (iD , ${ }^{1,2,3}$ Mélanie Escaich, ${ }^{3}$ Serge Bouznah, ${ }^{4}$ Clémence Rousselle, ${ }^{5}$ \\ Pascale De Lonlay, ${ }^{6,7}$ Pierre Canoui, ${ }^{8}$ Marie-Rose Moro, ${ }^{1,2,3}$ \\ Isabelle Durand-Zaleski (1) ${ }^{5,9,10}$
}

To cite: Lachal J, Escaich M, Bouznah S, et al. Transcultural mediation programme in a paediatric hospital in France: qualitative and quantitative study of participants' experience and impact on hospital costs. BMJ Open 2019;9:e032498. doi:10.1136/ bmjopen-2019-032498

- Prepublication history and additional material for this paper are available online. To view, please visit the journal (http:// dx.doi.org/10.1136/bmjopen2019-032498).

$\mathrm{JL}$ and ME contributed equally.

Received 29 June 2019 Revised 03 October 2019 Accepted 01 November 2019

A) Check for updates

(C) Author(s) (or their employer(s)) 2019. Re-use permitted under CC BY-NC. No commercial re-use. See rights and permissions. Published by BMJ.

For numbered affiliations see end of article.

Correspondence to Dr Jonathan Lachal; jonathan.lachal@gmail.com

\section{ABSTRACT}

Objective In France, immigrants with chronic diseases encounter numerous difficulties in gaining access to care and then in its initiation and organisation, difficulties only partly explained by socioeconomic factors. A transcultural mediation consultation programme has been set up in Necker Hospital in Paris to help families and professionals deal with these situations. The objective of this study was to assess the economic impact and the experience of this consultation.

Design Qualitative and quantitative descriptive study. Setting This study of inpatients treated at Necker Hospital included those whose families participated in transcultural mediation in 2014 through 2016.

Participants The first portion of the study applied quantitative methods and compared hospital costs before and after the mediation from the patients' records and accounting data for 15 cases. The qualitative portion analysed 15 semistructured interviews of physicians and families after the mediation, and a focus group of three psychologists.

Results The results show a systematic reduction in costs after mediation, associated with fewer emergency hospitalisations and the shift of care toward less specialised facilities, calmer relationships between families and professionals, improved mutual understanding and increased confidence by the professionals in the families' ability to manage the treatment.

Conclusion Transcultural mediation can benefit both patients and the healthcare system and may be useful for other hospitals that care for socially and culturally diverse patients.

\section{INTRODUCTION}

The high burden of chronic diseases-both communicable, such as HIV infection, and non-communicable, such as diabetes and kidney failure-is partially explained by socioeconomic factors. ${ }^{1-3}$ Immigrants face substantial delays in screening or in management after diagnosis, difficulties in access to care, ${ }^{4}$
Strengths and limitations of this study

The use of both qualitative and quantitative methods of study.

- Comparison of hospital costs before and after mediation, as noted in patients' records.

- Because the analysis was conducted at a single hospital, results cannot be generalised to other healthcare contexts.

by communication problems due to barriers that are both linguistic and cultural, ${ }^{56}$ and sometimes even by discrimination, ${ }^{78}$ which result in increased morbidity. ${ }^{3}$ To cope with these issues, many initiatives exist in health institutions, relying on language translators or cultural brokers. ${ }^{9}{ }^{10}$ Despite the fact that cultural brokers can be experts in the patient's culture, they are most of the time unable to understand the intricacies of the clinical language and 'medical world'. ${ }^{11}$

Babel Centre-European Resources Centre for Transcultural Issues-has been experimenting since 1998 with a new programme: transcultural mediation. This original programme is directed at hospital professionals dealing with difficult situations with their immigrant patients suffering from chronic diseases. ${ }^{12}$ Transcultural mediation is adapted from the cultural consultations that have been successfully used for mental health services, ${ }^{13-17}$ where a group of multicultural therapists are working with the concept of culture to allow migrant patients to rethink illness and care with their cultural theories. ${ }^{18}$ In the Babel Centre programme, a physician-mediator works together with a cultural broker (which we refer to as transcultural 
mediator), who is familiar with the patient's culture. This team meets with the patients, his or her family and the medical teams for a 2-hour consultation.

The objective of the transcultural mediation consultation is to modify the conditions of the interaction between the medical teams, on the one hand, and patients-and their families, as most of the time migrant patients' families are involved in medical decisions-on the other. It begins with the observation of a disconnect within the hospital between two explanatory models of disease. The medical model involves the search for a cause, based on concepts of evidence, while the explanatory model of patients and their families is based on a quest for meaning. ${ }^{19}$ Transcultural mediation aims to empower patients and their families by restoring the discourse of patients and their families and their own personal experience of the disease. The shared narrative of cultural and medical representations may allow each to find meaning in what goes on in treatment and to understand that the different discourses about the disease can coexist without one disqualifying the other. ${ }^{12}{ }^{20}$ As a result, patients and their families may become actors in the patient's recovery by mobilising resources from both the medical and cultural worlds. The medical teams should obtain keys to understanding the ways of thinking and behaving that come from other cultures. Finally, transcultural mediation promotes patient-doctor interaction and improves the therapeutic relationship. ${ }^{121}$

\section{METHODS}

Transcultural mediation, as performed in this programme that is part of the hospital's broader approach to a better reception of migrant patients, is a new type of consultation, and little is known about it. Therefore, we set out to explore the effect of a single mediation session on the participating medical staff and on patients and their families, as well as the actual impact on hospital costs.

Specifically, we wanted to describe both its processes and its outcomes. ${ }^{22}{ }^{23}$ Because the prospective payment system currently used to fund hospitals throughout the world cannot provide recurrent funding for cultural mediation, we wanted to document the impact on hospital resources use for hospital management.

\section{Setting}

Necker Children's Hospital (part of the Paris public hospital system, AP-HP) is a large paediatric and adult hospital in southern Paris. It serves as a reference centre for a number of rare metabolic disorders that cause both neuropsychiatric symptoms and psychological distress, as well as for HIV-infected children, adolescents and young adults. All of them need to follow a demanding treatment schedule and lifestyle changes, both of which can be hindered by cultural barriers. We included families of patients participating in transcultural mediation in 2014.

\section{Intervention}

The consultation for transcultural mediation lasts between 2 and 4 hours. It is organised at the request of a physician when the medical staff suspects that difficulties regarding a patient's treatment result from culturally related issues, that is, for example, treatment refusal from a lifethreatened migrant patient with no rational explanation of the refusal and a patient following a traditional treatment which may jeopardising the medical treatment. The mediation brings together the patient and his/her close family, the doctor and any member of the hospital staff involved in the treatment. The consultation is conducted by the mediation team, composed of a physician-mediator who is not a member of the medical unit, with a specific training in transcultural care (academic certification), and a transcultural mediator, a trained professional from the same culture as the patient's parents, sharing their native language and fluent in French.

In the first part of the transcultural mediation, the hospital physician is asked to describe the child's medical condition, the proposed treatment and the problems or concerns that led him/her to request the mediation. The cultural mediator translates, if necessary, and uses his/ her knowledge of the culture to make the medical statements accessible to the family. The physician-mediator, from the Babel Centre, reformulates some of the doctor's statements to ensure the family fully understands the issues related to their child's condition, and the cultural mediator translates if needed.

In the second part of the consultation, the family is invited to express, with the help of the cultural mediator, its own understanding of the origin of the disease and the treatments or protection possibilities available in the cultural area they come from.

Finally, with the help of the transcultural team, the family and the hospital medical staff are invited to build a story together, in which the disease finds a meaning in the child's life history and integrates simultaneously the resources of Western medicine and those of the family's culture of origin.

\section{Study and design sample}

Our study focused on inpatients at Necker Hospital who had participated in transcultural mediation in 2014. Participants included patients with HIV, rare metabolic diseases or neurodegenerative diseases.

We used two different samples. For the quantitative component, we included all patients referred to the transcultural consultation by the hospital paediatric subspecialists. Before the transcultural mediation programme was available, patients with metabolic disorders, neurodegenerative diseases and HIV infections were followed up in the appropriate specialised clinics and admitted either to day hospital departments or conventional wards whenever their condition deteriorated. After the mediation, follow-up took place either during hospital consultations or admissions, both of which were tracked in the hospital's computer system and the patients' charts. We chose a before/after design based on the analysis of claims data.

For the qualitative component, we used purposive sampling, selecting paediatric inpatients with different 
diseases and with different departments. In order to present the diversity of the clinical situations in which mediation has been offered at Necker, we asked both families and physicians present at one or more mediations to participate in this study. A focus group was also organised with three hospital psychologists.

The research team consisted of specialists in cultural mediation and researchers specialising in qualitative studies.

\section{Quantitative component \\ Data collection}

We estimated the cost of implanting the transcultural mediation at Necker Hospital and documented changes in monthly hospital resource use before and after the sessions took place. The cost of the intervention was estimated from the budget earmarked by the Regional Health Authority to cover its labour costs, including fees of the physician-mediator and the transcultural mediators. To this amount, we added the time cost of the hospital physicians who attended the consultations. We extracted from the hospital's discharge database all patient-linked admissions before and after the transcultural mediation took place. Hospital discharge data were mapped with clinical data, extracted manually from patients' charts; all personal identifiers were replaced by a study number. Unit costs were obtained from the hospitals' detailed accounting system. We did not include healthcare costs outside the hospital because the information was not recorded for the 'before' period. We did not include indirect costs. Because of the small size of the population and the heterogeneity in diseases and goals of the mediation, the before/after cost calculations are illustrative.

\section{Data analysis}

We estimated the average number of admissions, hospital days and costs per patient. Because the transcultural mediation became available in 2014-2015, the duration of the premediation period varied between patients, while the duration of the follow-up periods averaged 12 months. We calculated and averaged service use and cost per month for the before and after period by dividing the totals for each patient by the duration of follow-up.

\section{Qualitative component \\ Data collection}

We conducted two semidirective interviews for each situation: one with the doctor in charge and one with the patient and his family (together), accompanied, if necessary, by an interpreter. The interviews took place from 1 to 6 months after the mediation, depending on the participants' availability. We designed the semistructured interview guide, intended to focus on the relationship between the physician and the patient's parents before, during and after the mediation (box 1).

All interview subjects provided informed consent. The interviews took place in a medical office from July 2015 through July 2016. They lasted 1 hour and were conducted

\section{Box 1 Semistructured interview guide}

\section{Doctor's interview guide}

- Please describe the situation with the patient and his/her family before the mediation and what convinced you to ask for a transcultural mediation.

- Please tell me about the mediation.

- What did you learn that you didn't know about the patient?

- How is it going for your patient since the mediation?

- Please describe the relationship with your patient since the mediation.

What are your thoughts about the future?

\section{Parents' interview guide}

- Please tell me about your relationship with the doctor and the medical team before the mediation.

- Please tell me about the mediation.

- How is it going for you and your relative since the mediation?

- Please describe the relationship with your doctor since the mediation.

\section{Focus group interview guide}

- What did you know about transcultural mediation before participating with one of your patients?

- Please tell me about the mediation?

- How is this therapeutic setting different from usual care in your medical unit?

What do you think are the advantages and limits of the mediation?

by one researcher (ME). We also invited all health professionals to participate in interviews. However, only three psychologists from Necker Hospital accepted to participate. They requested to be interviewed together, so we conducted a focus group-type interview with them (box 1).

\section{Data analysis}

All interviews were audio-recorded and transcribed verbatim in French. Analysis of transcripts aimed at describing the main ideas and themes evoked by interviewees. ${ }^{24} 25$

The first stage of the analysis involved the interviewer (ME) checking the transcripts for accuracy against tape recordings. Then individual transcripts were repeatedly read and coded by three researchers (ME, JL and and M-RM) to identify initial themes, which were annotated in the margins. Each reading had the potential to bring up new insights. Recurrent themes (Morse \& Field, $1995)^{26}$ were then identified across transcripts; recurrent themes reflect a shared understanding of the phenomena in question among participants. This stage involved a more analytical ordering, as researchers tried to make sense of the connections between themes. Some of the themes tended to cluster. The process was dynamic and cyclic, with each transcript leading to the collection of further data and their subsequent analysis. The aim was to recognise ways in which narratives from the participants could be similar but also different. Researchers were in fact disciplined to discern returning patterns, but they also integrated new issues that emerged, so as to take account of convergences and divergences in the data. The last stage was to produce a coherent ordered table of 
the themes. The procedure for data analysis was inductive as the analysis of data from the literature was performed secondarily.

The three researchers who conducted the main analysis brought their results together and discussed them to improve the consistency and coherence of the analysis by ensuring that the themes identified were an accurate reflection of the data and that the analysis was not confined to one perspective. The results were discussed in repeated meetings with the entire research team. These discussions helped to highlight necessary clarifications or modifications among the themes identified. Validity was further enhanced by the care taken to distinguish clearly between what respondents said and researchers' interpretations or account of it. In the results, extracts of respondents' verbatim account have been chosen to exemplify the underlying recurrent themes. To protect confidentiality, identifying information has been removed in the quotes presented. The verbatim transcripts have been freely translated into English for the sole purposes of the present article, with attention paid to preserving the essential meaning and content, as well as, insofar as possible, general tone.

\section{Patient and public involvement}

Patients and the public were not involved in this study.

\section{RESULTS}

\section{Quantitative component}

Twenty-one patients and families were referred for transcultural mediation from January 2014 through December 2015. All 21 patients underwent the entire process, and 15 patients were analysed in full. The total cost of the mediation was estimated to be $€ 21600$ or roughly $€ 1000$ per patient. Hospital service use decreased after mediation for all patients, mostly explained by fewer admissions due to disease complications in HIV-infected patients, a shift from inpatient to consultation services for patients with metabolic disorders, or, for four patients with neurodegenerative disorders, admission to a specialised institution that had previously been rejected by the family (table 1 ).

\section{Qualitative component}

We included 15 interviews from nine doctors and six families, and three psychologists in the focus group. Two patients for whom transcultural mediation was conducted died a few weeks afterwards. For ethical reasons, we decided not to attempt to interview the family. Another family moved back to their home country before we were able to arrange an interview. Finally, for one patient, the situation remained very confrontational between the hospital and the family after the mediation, and the family and the doctor both refused to meet our researcher. Table 2 describes each family situation.

The results are organised around five themes: listen and be listened to, understand and be understood, trust and be trusted, make the most of everyone's resources and the limits of mediation (online supplemental digital content 1 (table) presents extra transcript excerpts exemplifying the results).

\section{Listen and be listened to}

The transcultural mediation session appeared to be an intense moment of communication that enabled the family to express itself about topics they have never previously mentioned.

Physician D - We've spoken a lot about the past and the family history, how the parents met, their marriage, and the father's refugee status in France, all things I didn't know. And for the practical aspects, the parents could say what they really expect in terms of care for their daughter.

The physicians thus had access to new information about the family history, information they had been unable to obtain in several months of consultations.

Table 1 Before-after comparison of hospital resources and hospital costs per patient per month

\begin{tabular}{|c|c|c|c|c|c|c|}
\hline Disease category & $\begin{array}{l}\text { Duration } \\
\text { of follow- } \\
\text { up before } \\
\text { mediation } \\
\text { (months) }\end{array}$ & $\begin{array}{l}\text { Duration } \\
\text { of follow- } \\
\text { up after } \\
\text { mediation } \\
\text { (months) }\end{array}$ & $\begin{array}{l}\text { Cost/month } \\
\text { before } \\
\text { mediation }(€)\end{array}$ & $\begin{array}{l}\text { Cost/ } \\
\text { month after } \\
\text { mediation }(€)\end{array}$ & $\begin{array}{l}\text { Hospital } \\
\text { days/month } \\
\text { before } \\
\text { mediation (n) }\end{array}$ & $\begin{array}{l}\text { Hospital } \\
\text { days/ } \\
\text { month after } \\
\text { mediation } \\
\text { (n) }\end{array}$ \\
\hline HIV infection $(n=3)$ & 32 & 7 & 1940 & 37 & 4 & 0 \\
\hline $\begin{array}{l}\text { Severe metabolic disorders } \\
\text { (leucinosis, isovaleric academia, } \\
\text { glycogenosis, citrullinemia, Wolman } \\
\text { disease }(n=7)\end{array}$ & 21 & 20 & 2297 & 776 & 4 & 1 \\
\hline $\begin{array}{l}\text { Mean for neurological and } \\
\text { neurodegenerative disorders (resistant } \\
\text { myoclonic-astatic epilepsy, febrile } \\
\text { infection-related epilepsy syndrome, } \\
\text { autism and mental retardation, } \\
\text { leucodystrophia) }(n=5)\end{array}$ & 19 & 11 & 3453 & 751 & 11 & 1 \\
\hline
\end{tabular}




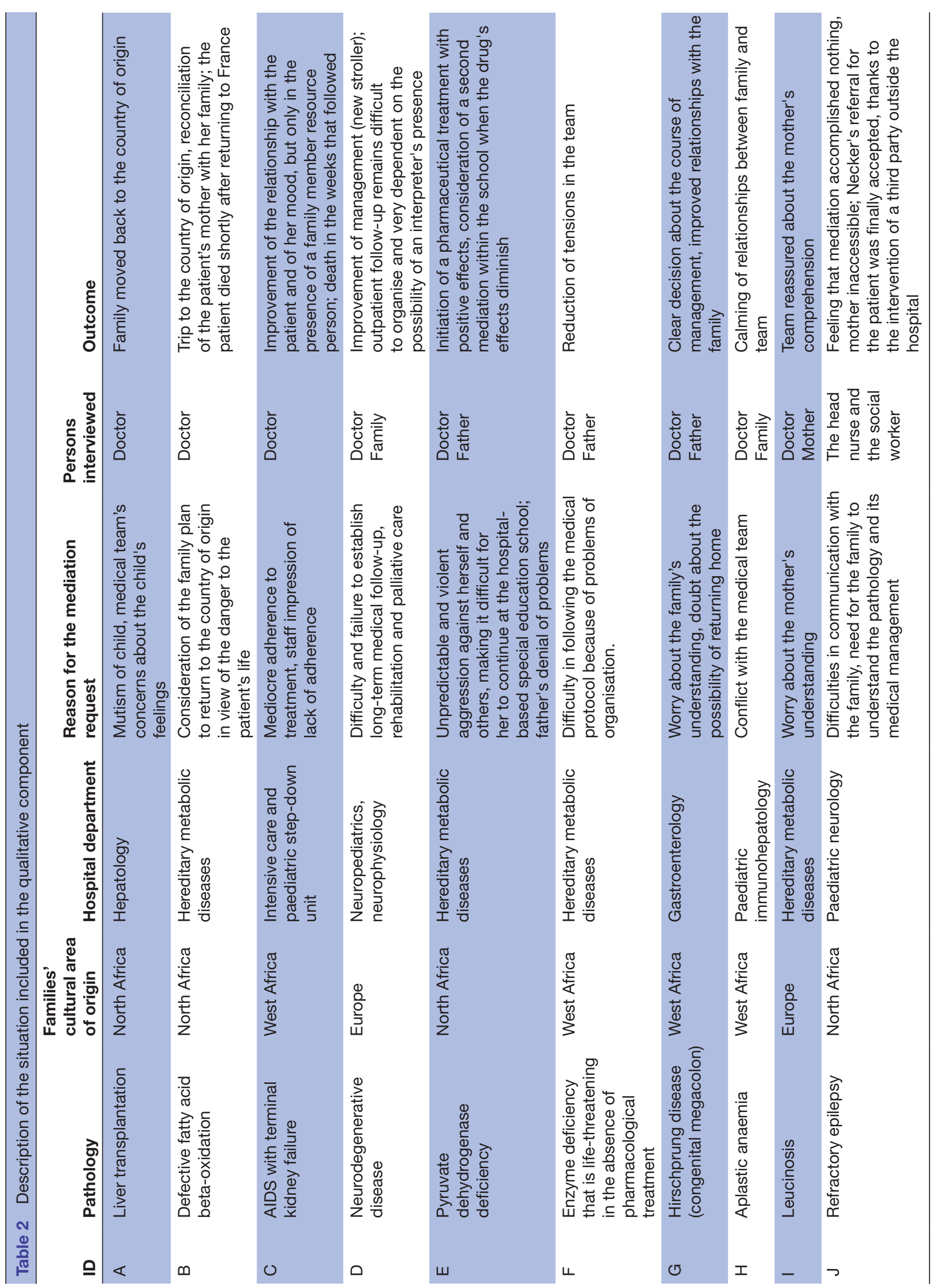


Physician C - We have discovered this extremely rich and complex world, and social interactions we don't know about.

When asked why they thought the families had not spoken about these subjects before, the doctors pointed out the lack of time they have to spend with one family, the language barrier and the resulting difficulties in communication, and their own discomfort in asking questions about the family's history. These questions appeared too intimate or private, as they go beyond the patient's medical condition.

Physician C - It surprised me that the doctor [from Babel Centre] was so directive in his way of asking questions, about this history which is very intimate, a bit shameful... and that it was perfectly accepted by the family. [...] The patient had been there for 3 months, and these were questions we didn't dare to ask, because it's a bit embarrassing.

Physician D - It's so private. I don't know whether this is our business, I am not sure it is my business to question his history. Do I have the right to ask this question?

The psychologists in the focus group considered that French doctors are reluctant to explore this kind of information related to the family history, that it is not part of their professional culture.

Focus Group - Before they didn't dare, they were afraid of being taken for yokels. The hospital, it's science triumphant, hyper-technological. When families talk about homeopathy, we snigger. It's two different universes. The families close up, stop talking, because they don't have the impression that the other is going to be able to listen to that. Not adhere, but just listen to this aspect.

Families are well aware of this and will generally not raise these topics by themselves. But the transcultural mediator induces a change in the relationship. His/her presence tells the family that the doctors are ready to listen to the family's point of view, placed in its historic and cultural context.

Focus Group - The mediator brings something from the native language that helps to touch deep inside. The patient experiences authenticity, is allowed to express emotions, and everyone begins to say things.

The focus group participants also mentioned that doctors must already deal with the intimacy of the human body. Moreover, they must often inflict some degree of violence on the body to extract the sickness. They need to maintain some distance to be able to stand this violence. Too much empathy may impair their ability to perform their task.

Focus Group - It's unbearable, the intimacy of others. The intimacy of bodies, one can disincarnate it, that's what medical school is for. To care for another and have to inflict violence to extract the disease from the body, you have to be able to cut off some amount of empathy, or it's impossible.

During the transcultural mediation, the doctor from Babel Centre (the physician-mediator) did not face the same issues. Moreover, the group discussion provides a setting that contains the intensity of emotion and feelings.

\section{Understand and be understood}

Both families and doctors pointed out the warm and thoughtful listening and mediation skills of the transcultural team from Babel Centre. The physician-mediator leads each to better understand the other. $\mathrm{He} /$ she supports the discourse of each participant in turn, and thus succeeds in creating bridges between families and the hospital team, so that each can both understand the other better and feel understood.

Mother, Family D - The doctor at the Babel Centre, he acted like a bridge between us so that we would understand each other better.

The families indicated especially that the mediation helped them to understand their relative's disease better.

Father Family H - The first hour we talked about the disease, and the doctor from Babel asked the same questions we asked when we arrived. And it was helpful, because we could finally understand everything, and the doctor asked the questions about the risks, which we didn't dare to ask, but I was happy he did, because now we understand it all.

The physicians reported that exploring the family's history and the situation around the patient's birth helped them to understand the origin of the difficulties they faced in providing medical care.

Physician E - I could finally understand what I hadn't until then. I had tried to figure out why this dad was so angry towards us, and why he said we were racists when he felt he was not understood. And it was his history that was still vivid inside him, the fact he still suffered from the distressing circumstances of his arrival in France.

The families were aware of the effort the physicians make to understand them, and they wanted, beyond the doctors' purely medical skills, which they never challenged, for the physicians to be able to understand the difficulty of their daily life with a severely ill patient.

Mother, Family D - The doctor, he understood us that day, he understood what we were saying, because he had the translation, because it's complicated to have a sick child, to have someone who is sick. The visits, it happens here, and once it's over, everyone goes home. But us, we stay with the disease, and it's very hard. 
In turn, the physicians explained how important it is for them to be understood by the families, particularly about the healing intentions behind sometimes violent medical acts.

Physician A - It was important to me that they understand it [everything that we did to the patient] was not directed towards her, but towards her sickness.

Physician B - Sometimes we're rude, we're crude, but we don't want to be rude, we don't want to be crude, the doctor from Babel Centre was able to translate our intention and to express the fact that we just want the patient to get better.

The mediation work performed by the transcultural team goes beyond the simple translation of words; it allows the participants to gain access to the feelings, representations, and intentions behind behaviours. Behaviour that previously appeared puzzling and sometimes threatening therefore became meaningful to the other party. The members of the focus group concluded that the mediation brings participants to see beyond the professional and social identities imposed by the situation and makes it possible to have a discussion between people, between humans.

Focus group - The mediation helps human beings to meet each other, while the hypertechnological blocks that. It reintroduces equality; there's no more hegemony, we are all humans.

\section{Trust and be trusted}

The parents' discourse about their life informed the physicians about the social, familial, and cultural resources available outside the hospital. Moreover, being able to talk in their native language allowed them to express the full complexity of their thoughts. They appeared less vulnerable and isolated.

Physician B - Eventually, with the mediation we realized she was a really good mother, that she cared about her child, his history. And the little things aside where she was not stimulating him, it was because we hadn't explained to her, but she was really willing to do things well. I didn't have any more doubts about this mother's intentions, about whether or not she understood.

In some cases, doctors stated that they felt more confident in the family's ability to handle their relative's condition and treatment effectively after the mediation. It changed their vision of the family's reliability.

Physician I - I will say there is more trust now, because before there were times when they missed some appointments, when some exams were not done, so it was a bit difficult. For me there is more trust from both sides.

\section{Make the most of everyone's resources}

One of the objectives of the mediation is to be able to bring the patients' families and the physicians together in a joint plan for the future, which can combine both the resources of European medicine and those available to patients and their families. The different protagonists in the mediation participate in building this plan, assisted by the transcultural team.

Physician B - We went from a situation where we were wondering if we'd made the right decision, to mutual understanding and we had really worked together. Everything was flowing, as if we were talking to ourselves finally, because it was truly obvious for both sides. When I say, as if we were talking to ourselves, I mean, that we understood each other so well that it was as if we had talked to ourselves with our own reasoning.

The physician-mediator is then responsible for creating synergy between the medical world and the world of the patients and families.

Physician C - And then, at the end, the physicianmediator, and for us, that was also something important, repeated the association between invasive medicine, the technique we use, and then, the whole cultural context and all these beliefs that are very important for (the adolescent) and her grandmother. So he brought back the coherence of all that, the fact that we were all there to protect (her) at the same time, we, her grandmother, and the more animist part of the family, through talismans or protection.

Nonetheless the integration of medical discourse into the patient's world does not always take place. Suggestions are then made to each protagonist: the family and the medical team. These proposals enable progress, but they are not put together in the physician's discourse into something that could constitute a joint project, mobilising the resources of each participant into the higher objective of the patient's best interest.

Physician $\mathrm{H}$ - The end of the mediation was especially a dialogue between the physician-mediator and the mother, it covered the entire family framework and structure, ethnicities, but well, most of the group were just spectators.

We thus identified two types of interaction between the mediation's protagonists. In one case, each receives information and a proposal for him or herself, for his or her side. Moreover, sometimes, sharing of information leads to the possibility of developing a joint project.

\section{The limits of mediation}

When asked if the relationship to their doctor and the hospital staff as a whole had changed after the mediation, the families expressed their trust in their doctor's skills but said nothing had changed for them in terms of the relationship. They underlined that an interpreter should 
be present for all consultations if needed, and that the listening and communication skills of the hospital staff need improvement.

Mother Family D - Nothing has changed. Our problem is the language. We need an interpreter. We would really like to understand him [the doctor]. But when he acts like that, we think he doesn't respect us. Our problem is that we don't understand each other, he doesn't understand us, we feel he doesn't make efforts to cure the disease, we have this feeling. He is a very good doctor, but we want him to do something against the disease.

Father Family F - My thoughts haven't changed. The doctor is wonderful and so is the team. But there are other people to whom it is difficult to explain things.

The physicians also described the difficulties they experienced concerning situations involving daily care for the patient, right after the mediation. They felt left alone again without the transcultural team's help and lost about how to continue the work initiated during the mediation.

Physician C - Right after the mediation we felt really frustrated. The mediation had opened a lot of doors, but after it we were alone, we didn't know what to do. We thought: the mediator will not come back, we have to find the solution by ourselves.

Moreover, some doctors appeared puzzled by the impression that the family was still not convinced after the mediation that the hospital's diagnosis of the patient was 'right'. This question was raised during the focus group, and the participants confirmed it is difficult for doctors to stop trying to convince the families of the veracity of their medical conclusions.

Focus Group - Even though the physicians want to understand what is going on for the other, the objective nonetheless is that the families adhere to their treatment, in the doctors' own world. It's only when the issue no longer has to do with treatment or cure that they can agree to let go of something and enter into another dimension besides their own.

It thus appears that while transcultural mediation broadens physicians' views about the explanatory models that make sense to their patients' families, they still need the help of a person specifically trained in transcultural care to handle complex, culturally related situations.

\section{DISCUSSION}

We estimated that our mediation programme costs about $€ 1000$ per patient. It would be useful to know whether these costs are offset by more rational healthcare use, and we did document some reduction in healthcare costs after mediation, but our study design does not allow us to determine whether the reduction is due to mediation or other, unrelated factors.
The qualitative component revealed the staff's newly gained confidence in the families' ability to manage the disease and in part by better adherence to the treatment schedules and care management, which may explain the shift from inpatient to outpatient settings. We cannot exclude, however, that mediation was more likely to be requested during a period of particularly intensive medical care and that remission could follow independently from the mediation. The patient's conditions, however, were chronic and severe, with recurring acute episodes which would be captured during the follow-up period of 7-20months, and these preliminary observations would support the continuation of the programme and data collection in negotiations with the hospital management.

The analysis of the interviews showed that the mediation enabled participants to become open to and more aware of the logic of the other's thoughts. The physicians are now better able to envision the singularity of the family's functioning and the importance of giving a meaning to the disease above and beyond biomedical Cartesian logic. This opening makes physicians more aware of and sensitive to the experience of immigrant families. Nonetheless, it does not modify their conception of medicine and the practices associated with it. The physicians are satisfied to have gained access to the families' representations of the disease. They understand better how the family functions in dealing with the patient's disease. However, the compromise is always fragile, and the desire to convince the family of the validity of European medicine remains a concern.

The results underline the value of mediation in calming the relationship and making healthcare providers more aware of the issue of cultural otherness. Nonetheless, its objective is not to transform any of the protagonists in depth. This obvious limitation of mediation is nonetheless intrinsic to its objectives: overcome a barrier, remobilise the care and recommit to the therapeutic relationship. The brevity of one or even several mediation sessions means it cannot lead to deep changes in how either protagonist functions. This is an inherent limitation of all short-term therapy: it can act immediately on the symptoms but does not induce long-term changes in how a person functions. ${ }^{27}$ For some patients, therefore, mediation cannot replace transcultural psychotherapeutic care when it is necessary. Similarly, it does not render the professionals autonomous in handling new obstacles or a new situation with this family or others. It will then be necessary to have recourse again to mediation, as foreseen in the protocol.

In the interviews, the physicians questioned their right to enter into the private life of the families. Within the hospital, the body is not the site of intimacy but the object of care. Inversely, the narrative of the family history, the meaning of the onset of the disease and its impact on the psychological life are all aspects that, in theory, do not concern either the diseased body or its care. These questions are thus as a general rule either delegated to 
specialists of the mind (psychologists or psychiatrists) or returned back to the private sphere.

Several factors feed this disconnect. On the one hand, the organisation of French medical training has traditionally focused on the acquisition of treatment techniques, and its continued movement toward hyperspecialisation contributes to the disembodiment of ill health. On the other hand, somatic care sometimes entails some amount of violence to the patient's body, especially when the patient is a child. The physicians' reluctance to explore the intimacy of families can thus be understood as a method of emotional detachment to protect themselves and the ongoing course of treatment. The transcultural mediation sessions plunge physicians into a universe of multiple meanings through a procedure unusual for them. Following this procedure can be disturbing for some.

It is often difficult for the medical team to return to these questions after the mediation, without the presence of the transcultural team. The mediation thus permits a compromise. The acceptance of what the families have to say and the entry into their private lives make it possible to restore the relationship with them. However, the sharing of this privacy is compartmentalised and limited to the space of the group and the time of the mediation. The space of somatic care is thus preserved as well.

The quantitative approach should be considered exploratory. Disease groups and follow-up periods varied widely, and mediation occurred at diverse moments along the treatment pathway. The sample size was small, with complete data available for 15 of the 21 patients; the others had died or returned to their country of origin. Our analysis was also restricted to acute hospital care. We did not measure the costs of shifts to family care or to specialised institutions. We fully acknowledge that the transfer from hospital care to consultations or specialised institutions is not necessarily a net savings for the healthcare system. We would like, however, to stress that a highly specialised children's hospital like Necker is better suited for the diagnosis and initial treatment of complex disorders, while other, less technical settings can provide adequate follow-up for stable patients. Our perspective was that acute hospitals should invest in cultural mediation insofar as it helps to reduce avoidable admissions and improve quality of care.

The qualitative interviews were conducted at only one hospital, and they cannot be generalised to all care situations. That the participating families were principally from Africa (North Africa and sub-Saharan Africa) and Europe also limits the possibility of generalisation. This population is, however, consistent with the composition of immigration into France. ${ }^{28}$ The very diverse cultural and medical contexts we studied nonetheless enabled us to envision a certain degree of transposition of our results to other contexts of care in France and internationally.

\section{CONCLUSION}

The difficulties encountered in providing care for migrant families have a major cost in terms of both morbidity and healthcare finances. Transcultural mediation has been proven to be a simple and effective tool for reducing barriers in care and reducing the cost. It does not modify medical practices profoundly, but it does authorise an encounter between two worlds, an encounter that calms the relationship and results in a resumption of stalled care. In our opinion, this type of programme should be widely developed among hospital groups that treat immigrants. We recommend that one physician-mediator be trained in every hospital centre in complement to existing interpreters' training. The training should include transcultural approaches, concepts of culture and effective group work. Then, work to make the entire medical staff aware of this programme should be sufficient to enable them to identify cases for which mediation is indicated and to call in the mediation team. The training of one physicianmediator per centre thus appears to be the most rational and efficient method. Moreover, the specialisation of some physicians in transcultural mediation is consistent with the development in recent years of new disciplines within the medical profession, as in the treatment of pain and palliative care. ${ }^{29} 30$ These new disciplines, which now play an important role in care, make it possible to build bridges between the increasing specialisation of medical techniques and the development of patient-centred care.

\section{Author affiliations}

${ }^{1}$ AP-HP, Cochin Hospital, Maison de Solenn, Paris, France

${ }^{2}$ Université de Paris, PCPP, Boulogne-Billancourt, France

${ }^{3}$ CESP, Fac. de médecine - Univ. Paris-Sud, Fac. de médecine - UVSQ, INSERM,

Université Paris-Saclay, Villejuif, France

${ }^{4}$ Centre Babel, European Resource Center in Transcultural Medicine, Paris, France

${ }^{5}$ URCEco lle de France, Hôpital de l'Hôtel Dieu, Paris, France

${ }^{6}$ Department of Pediatrics, Hôpital des Enfants Malades, APHP, Université Paris

Descartes, Paris, France

${ }^{7}$ INSERM, U393, Paris, France

${ }^{8}$ Department of Child and Adolescent Psychiatry, Hôpital des Enfants Malades, APHP, Paris, France

${ }^{9}$ AP-HP Santé Publique, Hôpital Henri Mondor, Créteil, France

${ }^{10}$ ECEVE - UMR1123, INSERM, Paris, France

Acknowledgements We thank the Babel Centre, Inserm 1178 (CESP), Necker Children's Hospital (AP-HP) and the lle-de-France regional health agency for their support of this research; Jo Ann Cahn for the translation; and all the investigators for their participation and help: Pr Stéphane Blanche, Department of Pediatric Immuno-Hematology, Hôpital Necker-Enfants Malades, AP-HP, France; Dr Jean Baptiste Arnoux, Manuella Bayard and Valérie Barbier, Department of Inherited Metabolic Diseases, Hôpital Necker-Enfants Malades, APHP; Pr Bernard Golse and Dr Laure Woestelandt, Department of Child and Adolescent Psychiatry, Hôpital des Enfants Malades, APHP, Paris France.

Collaborators Stéphane Blanche, Jean Baptiste Arnoux, Manuella Bayard, Valérie Barbier, Bernard Golse, Laure Woestelandt.

Contributors JL: conceptualisation, methodology, supervision,validation, writing and original draft preparation, writing of review and Editing. ME: conceptualisation, methodology, investigation, writing and original draft preparation, writing of review and editing. SB: conceptualisation, methodology, investigation, writing and original draft preparation, writing of review and editing. CR: conceptualisation, methodology, investigation, writing and original draft preparation. PDL: investigation, supervision, writing and original draft preparation. PC: investigation, supervision, writing and original draft preparation. M-RM: conceptualisation, methodology, supervision, 
validation, writing and original draft preparation. ID-Z: conceptualisation, methodology, supervision, validation, writing and original draft preparation, writing of review and editing.

Funding This work was supported by lle-de-France regional health agency. The funders had no role in study design, data collection and analysis, decision to publish, or preparation of the manuscript.

Competing interests None declared.

Patient consent for publication Not required.

Ethics approval Written consent was collected, and ethical committee approval was not required for qualitative or retrospective exploration at the time of the inclusions.

Provenance and peer review Not commissioned; externally peer reviewed.

Data availability statement All data relevant to the study are included in the article or uploaded as supplementary information.

Open access This is an open access article distributed in accordance with the Creative Commons Attribution Non Commercial (CC BY-NC 4.0) license, which permits others to distribute, remix, adapt, build upon this work non-commercially, and license their derivative works on different terms, provided the original work is properly cited, appropriate credit is given, any changes made indicated, and the use is non-commercial. See: http://creativecommons.org/licenses/by-nc/4.0/.

\section{ORCID iDs}

Jonathan Lachal http://orcid.org/0000-0002-6282-7359

Isabelle Durand-Zaleski http://orcid.org/0000-0002-4078-1476

\section{REFERENCES}

1 Larchanché S. Intangible obstacles: health implications of stigmatization, structural violence, and fear among undocumented immigrants in France. Soc Sci Med 2012;74:858-63.

2 de Waure C, Bruno S, Furia G, et al. Health inequalities: an analysis of hospitalizations with respect to migrant status, gender and geographical area. BMC Int Health Hum Rights 2015;15:2.

3 Makowski AC, Kofahl C. Benefit and adherence of the disease management program "diabetes 2": a comparison of Turkish immigrants and German natives with diabetes. Int J Environ Res Public Health 2014;11:9723-38.

4 De Luca G, Ponzo M, Andrés AR. Health care utilization by immigrants in Italy. Int J Health Care Finance Econ 2013;13:1-31.

5 Bermejo I, Hölzel LP, Kriston L, et al. [Barriers in the attendance of health care interventions by immigrants]. Bundesgesundheitsblatt Gesundheitsforschung Gesundheitsschutz 2012;55:944-53.

6 Mirza M, Luna R, Mathews B, et al. Barriers to healthcare access among refugees with disabilities and chronic health conditions resettled in the US Midwest. J Immigr Minor Health 2014;16:733-42.

7 Tjaden LA, Noordzij M, van Stralen KJ, et al. Racial disparities in access to and outcomes of kidney transplantation in children, adolescents, and young adults: results from the ESPN/ERAEDTA (European Society of pediatric Nephrology/European renal Association-European dialysis and transplant association) registry. Am J Kidney Dis 2016;67:293-301.

8 Carde E. Les discriminations selon l'origine dans l'accès aux soins. Santé Publique 2007;19.

9 National Center for Cultural Competence,. Bridging the Cultural Divide in Health Care Settings: The Essential Role of Cultural Broker Programs [Internet]. Georgetown: U.S. Department of Health and
Human Services, 2004. Available: https://nccc.georgetown.edu/ culturalbroker/Cultural_Broker_EN.pdf [Accessed cited 2018 Jul 4].

10 Torres S, Labonté R, Spitzer D, et al. Improving health equity: the promising role of community health workers in Canada. Hcpol 2014;10:73-85.

11 Bouznah S, Larchanché S. Transcultural mediation in the management of cancer patients in the tropical area. Tropical HematoOncology 2015:55-64.

12 Bouznah S, Larchanché S, Droz J-P, et al. Transcultural Mediation in the Management of Cancer Patients in the Tropical Area. In: Tropical hemato-oncology. Cham: Springer International Publishing, 2015: 55-64

13 Bhui KS, Owiti JA, Palinski A, et al. A cultural consultation service in East London: experiences and outcomes from implementation of an innovative service. Int Rev Psychiatry 2015;27:11-22.

14 Tarricone I, Atti AR, Braca M, et al. Migrants referring to the bologna transcultural psychiatric team: reasons for drop-out. Int J Soc Psychiatry 2011;57:627-30

15 Kirmayer LJ, Rousseau C, Rosenberg E, et al. Development and Evaluation of a Cultural Consultation Service in Mental Health [Internet. Montreal: Mc Gill University, 2001. https://www.mcgill.ca/ tcpsych/files/tcpsych/Report11.pdf

16 Kirmayer LJ, Groleau D, Guzder J, et al. Cultural consultation: a model of mental health service for multicultural societies. Can $J$ Psychiatry 2003;48:145-53.

17 Owiti JA, Ajaz A, Ascoli M, et al. Cultural consultation as a model for training multidisciplinary mental healthcare professionals in cultural competence skills: preliminary results. J Psychiatr Ment Health Nurs 2014:21:814-26.

18 Sturm G, Heidenreich F, Moro M. Transcultural clinical work with immigrants, asylum seekers and refugees at Avicenne Hospital, France. Int J Migr Health Soc Care 2009;4:33-40.

19 Good BJ. Medicine, Rationality and Experience: An Anthropological Perspective [Internet]. Cambridge University Press; 1994. (Lewis Henry Morgan Lectures). Available: https://books.google.fr/books? id=p7-Enmqb604C

20 Bouznah S, Lewertowski C, Margot-Duclot A. Intérêt d'un dispositif de médiation interculturelle dans la prise en charge hospitalière des patients migrants douloureux chroniques. Douleurs 2003;4:123-33.

21 Es-Safi L. La médiation culturelle dans les hôpitaux ouComment rétablir la communication entre les patients d'origine étrangère et le personnel soignant. Pensée Plurielle 2001:1:27-34.

22 Curry L, Nunez-Smith M. Mixed methods in health sciences research: a practical primer. Los Angeles: SAGE (SAGE mixed methods research series), 2015: 394

23 Creswell JW, Klassen AC, Clark VLP, et al. Office of Behavioral and Social Sciences Research. Mixed Methods Research - Office of Behavioral and Social Sciences Research [Internet]. National Institutes of Health 2011

24 Bradshaw C, Atkinson S, Doody O. Employing a qualitative description approach in health care research. Glob Qual Nurs Res 2017;4.

25 Sandelowski M. Whatever happened to qualitative description? Res Nurs Health 2000;23:334-40.

26 Morse M, Field M. Qualitative research methods for health professionals. SAGE 1995:272.

27 Lindfors $\mathrm{O}$, Knekt P, Heinonen E, et al. The effectiveness of shortand long-term psychotherapy on personality functioning during a 5-year follow-up. J Affect Disord 2015;173:31-8.

28 Insee. Institut national de la Statistique et des Etudes Economiques (INSEE). Etrangers-Immigrés. Paris: Insee, 2016.

29 Powell RA, Mwangi-Powell FN, Radbruch L, et al. Putting palliative care on the global health agenda. Lancet Oncol 2015;16:131-3.

30 The Lancet. Palliative care: a peaceful, humane global campaign is needed. Lancet 2014;383:487. 
Correction: Transcultural mediation programme in a paediatric hospital in France: qualitative and quantitative study of participants' experience and impact on hospital costs

Lachal J, Escaich M, Bouznah S, et al. Transcultural mediation programme in a paediatric hospital in France: qualitative and quantitative study of participants' experience and impact on hospital costs. BMJ Open 2019;9:e032498.

The name of the co-author of this paper was misspelt. Clémence Rousselle is the correct name.

Open access This is an open access article distributed in accordance with the Creative Commons Attribution Non Commercial (CC BY-NC 4.0) license, which permits others to distribute, remix, adapt, build upon this work non-commercially, and license their derivative works on different terms, provided the original work is properly cited, appropriate credit is given, any changes made indicated, and the use is non-commercial. See: http://creativecommons.org/licenses/by-nc/4.0/.

C Author(s) (or their employer(s)) 2020. Re-use permitted under CC BY-NC. No commercial re-use. See rights and permissions. Published by BMJ.

BMJ Open 2020;10:e032498corr1. doi:10.1136/bmjopen-2019-032498corr1

(A) Check for updates 